\title{
PERDA DENTÁRIA EM ADOLESCENTES DE UMA INSTITUIÇÃO PÚBLICA
}

\author{
DENTAL LOSS IN ADOLESCENTS FROM A PUBLIC INSTITUTION \\ PÉRDIDA DE DIENTES EN ADOLESCENTES DE UMA INSTITUCIÓN PÚBLICA
}

Ana Carla Rodrigues de Castro ${ }^{1}$, Taynara Vieira Carneiro², Vanara Florêncio Passos ${ }^{3}$, Regina Glaucia Lucena Aguiar Ferreira ${ }^{4}$

\section{RESUMO}

Descrever a prevalência da perda dentária em adolescentes de Fortaleza, Ceará. Pesquisa descritiva, observacional, transversal, de natureza quantitativa, tendo como população de estudo 59 adolescentes de uma escola pública. Os dados foram coletados mediante exames bucais e questionário sobre dados demográficos, histórico ortodôntico e hábitos de higiene bucal. Posteriormente, foram tabulados no Microsoft Excel 2010 e analisados estatisticamente pelo software SPSS 22.0. Foram empregados os testes "Qui-quadrado de Pearson" e "Exato de Fisher", com o nível de significância de $5 \%$. A prevalência de perda dentária foi de $28,8 \%$, não estando associada ao sexo ou frequência de escovação. Os segundos molares superiores foram os mais afetados (16,67\% e 13,3\%, respectivamente). A prevalência de perda dentária entre os adolescentes mostrou-se elevada, comparada aos resultados do último levantamento epidemiológico nacional, o que remete à necessidade de se implementarem políticas públicas de saúde que contribuam para modificar esta realidade.

Palavras-Chave: Perda de dente; Adolescente; Saúde bucal.

\section{ABSTRACT}

Describe the prevalence of tooth loss in adolescents in the city of Fortaleza, Ceará. This is a descriptive, observational, cross-sectional, quantitative study, having as its population 59 adolescents from a public school. Data were collected by means of oral exams and questionnaire, tabulated in Microsoft Excel 2010 and statistically analyzed using SPSS 22.0 software. Pearson's chi-square and Fisher's exact tests were used with a 5\% significance level. The prevalence of tooth loss was $28.8 \%$, not being associated with gender or brushing frequency. The maxillary second molars were the most affected (16.67\% and $13.3 \%$, respectively). The prevalence of tooth loss was high when compared to the last national epidemiological survey. Public health policies that contribute to changing this reality are needed.

Keywords: Tooth loss; Adolescent; Oral health.

\section{RESUMEN}

Describir la prevalencia de la pérdida dentaria en adolescentes de la ciudad de Fortaleza (Ceará). Investigación descriptiva, observacional, transversal y cuantitativa, que tuvo como población de estudio 59 adolescentes de una escuela pública. Los datos fueron obtenidos a través de exámenes bucales y cuestionario, tabulados en Microsoft Excel 2010 y analizados estadísticamente utilizando el software SPSS 22.0. Se emplearon las pruebas de chi-cuadrado de Pearson y exacta de Fisher, con el nivel de significación del 5\%. La prevalencia de pérdida dentaria fue del 28,8\%, sin asociación con el sexo o la frecuencia de cepillado. Los segundos molares superiores fueron los más afectados (el 16,67\% y el 13,3\%, respectivamente). En suma, la prevalencia de pérdida dentaria se reveló elevada, en comparación con la última encuesta epidemiológica nacional. Se necesitan políticas de salud pública que contribuyan a cambiar esta realidad.

Palabras Clave Pérdida dentaria; Adolescente; Salud bucal.

\footnotetext{
${ }^{1}$ Universidade Federal do Ceará, Fortaleza, Brasil. (0000-0001-7840-7394)

2 Universidade Federal do Ceará, Fortaleza, Brasil. (0000-0002-9235-6711)

${ }^{3}$ Universidade Federal do Ceará, Fortaleza, Brasil. (0000-0001-5121-0436)

${ }^{4}$ Universidade Federal do Ceará, Fortaleza, Brasil. (0000-0003-4225-7958)
} 


\section{INTRODUÇÃO}

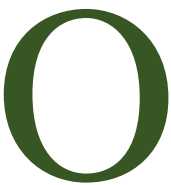

s efeitos adversos da cárie dentária podem interferir negativamente no desenvolvimento das crianças e dos adolescentes, bem como no desempenho de suas atividades cotidianas. A dor, as infecções, as perdas dentárias precoces e os distúrbios de ordem mastigatória podem ocasionar danos estéticos, funcionais, psicológicos e sociais ${ }^{1,2}$.

Durante a adolescência, estabelecem-se comportamentos e estilos de vida que poderão influenciar o padrão de morbidade e de cuidados de saúde futuros ${ }^{3}$. É nessa fase que a cárie dentária, os traumatismos dentários e a doença periodontal, em especial a gengivite, têm apresentado prevalências significantes ${ }^{4}$.

De acordo com o levantamento epidemiológico nacional de saúde bucal, o SB BRASIL $2010^{5}$, houve marcante redução nas perdas dentárias (prevalência de $17,4 \%$ ) quando comparadas aos 38,9\% registrados no levantamento epidemiológico de 200. Tal redução se deve às mudanças nos critérios de identificação da cárie dentária, além de medidas ou fatores de proteção, como a expansão da fluoretação das águas de abastecimento público e a introdução do dentifrício fluoretado no mercado, entre outros fatores ${ }^{2,6,7}$.

A meta estabelecida pela Organização Mundial da Saúde $(\mathrm{OMS})^{8}$ para jovens com 18 anos de idade, para o ano 2000, é que $85 \%$ dos indivíduos deveriam ter todos os dentes presentes na boca, entretanto, Silveira e colaboradores ${ }^{9}$ constataram que $83,7 \%$ dos adolescentes apresentavam todos os dentes presentes na boca, percentual inferior àquele estabelecido pela OMS.

Mesmo sendo considerada um problema de saúde pública, a perda dentária tem sido pouco investigada entre adolescentes brasileiros. O objetivo deste estudo foi descrever a prevalência da perda dentária em adolescentes de uma instituição pública de ensino, no município de Fortaleza, Ceará.

\section{METODOLOGIA}

Trata-se de uma pesquisa descritiva, observacional, transversal, de natureza quantitativa, tendo como população de estudo alunos da Escola Municipal Monsenhor Linhares, localizada no bairro Amadeu Furtado, município de Fortaleza, Ceará.

De uma população de 82 estudantes regularmente matriculados e cursando o $9^{\circ}$ Ano do Ensino Fundamental no ano de 2018 (critério de inclusão), obteve-se uma amostra composta por 59 adolescentes, cujos pais ou responsáveis concordaram com sua participação na pesquisa. É importante esclarecer que houve demora em se obter a aprovação junto ao Comitê de Ética em Pesquisa da Universidade Federal do Ceará, e, por conseguinte, a coleta dos dados só foi possível se dar no período de março a maio de 2019. Ressaltase que a população de estudo foi preservada (alunos que cursaram o $9^{\circ}$ ano em 2018). Não participaram da pesquisa 23 estudantes, sendo 21 por recusa ou desinteresse de pais/responsáveis em assinar o Termo de Consentimento Livre e Esclarecido e dois por terem sido selecionados para participar do préteste. Este último foi realizado no intuito de se aprimorar o instrumento de coleta de dados.

As informações foram obtidas por meio de exames bucais e da aplicação de questionário estruturado. Os exames foram realizados por uma única pesquisadora, em uma sala da escola, sob luz ambiente, utilizando máscara, luvas e espátulas de madeira. Enquanto a pesquisadora principal examinava os estudantes, a auxiliar preenchia o questionário estruturado, incluindo dados demográficos, histórico ortodôntico e hábitos de higiene bucal. O questionário foi elaborado tomando como base outros instrumentos, publicados na literatura pertinente.

Os dados foram tabulados no Microsoft Excel 2010 e analisados estatisticamente por meio do software SPSS 22.0 para Windows (SPSS Inc., Chicago IL, USA). Na análise, foram empregados os testes "Qui-quadrado de Pearson" e "Exato de Fisher", adotando o nível de significância de 5\% $(\mathrm{p}<0.05)$.

Seguindo-se os preceitos da Resolução CNS $\mathrm{N}^{\mathrm{o}} 466$, de 12 de dezembro de 2012, que rege as 
pesquisas com seres humanos, submeteu-se o estudo ao Comitê de Ética em Pesquisa da Universidade Federal do Ceará, tendo sido aprovado sob o Parecer No 2.607.104.

\section{RESULTADOS}

Entre os 59 adolescentes participantes do estudo, 29 eram do sexo masculino $(49,2 \%)$ e 30 do sexo feminino $(50,8 \%)$, com idade entre 13 e 17 anos, como pode ser visto na Tabela 1.

Tabela 1 - Perfil sociodemográfico dos estudantes e fatores comportamentais relacionados à saúde bucal. Fortaleza $(\mathrm{CE})$, maio de 2019.

\begin{tabular}{ccc}
\hline Higiene oral & $\mathbf{N}$ & $\mathbf{\%}$ \\
\hline Idade & 1 & \\
13 anos & 12 & $1,69 \%$ \\
14 anos & 34 & $20,34 \%$ \\
15 anos & 8 & $57,63 \%$ \\
16 anos & 3 & $13,56 \%$ \\
17 anos & 59 & $5,09 \%$ \\
Total & $100 \%$ \\
Etnia & 12 & \\
Negro & 11 & $20,3 \%$ \\
Branco & 2 & $18,7 \%$ \\
Amarelo & 34 & $3,4 \%$ \\
Pardo & 59 & $57,6 \%$ \\
Total & $100 \%$ \\
& Uso do fio dental & \\
Sim & 20 & $33,9 \%$ \\
Não & 39 & $66,1 \%$ \\
Total & 59 & $100 \%$ \\
Frequência do uso do fio dental & \\
Todos os dias & 5 & $8,47 \%$ \\
Alguns dias da semana & 6 & $10,17 \%$ \\
Uma vez por semana & 2 & $3,39 \%$ \\
Sem regularidade & 7 & $11,87 \%$ \\
Não respondeu & 29 & $66,1 \%$ \\
Total & 59 & $100 \%$ \\
Extraiu devido a tratamento ortodôntico \\
Sim & 2 & $3,4 \%$ \\
Não & 57 & $96,6 \%$ \\
Total & 59 & $100 \%$ \\
\hline
\end{tabular}

Fonte - Produzida pelos Autores.

No que diz respeito à quantidade de dentes ausentes, constatou-se que, entre os 59 estudantes, 30 unidades dentárias foram perdidas (o que correspondeu a uma média de 0,51 dentes perdidos por indivíduo), com uma variação de 1 a 7 dentes perdidos, por estudante. Dentre as causas que levaram à perda dentária, a cárie foi o motivo mais relatado pelos adolescentes $(83,33 \%)$, tendo a extração por motivos ortodônticos correspondido a 16,67\%.
Dentre as unidades dentárias perdidas, os segundos molares superiores do lado direito (Dente 17 ) foram os mais prevalentes $(16,67 \%)$.

Observou-se uma prevalência de $28,8 \%$ de dentes perdidos, sendo o maior percentual entre as adolescentes (18,6\%), não havendo, entretanto, significância estatística nessa associação (Tabela 2).

Tabela 2 - Associação entre perda dentária e sexo entre os estudantes da Escola Monsenhor Linhares. Fortaleza (CE), maio de 2019.

\begin{tabular}{cccc}
\hline Variáveis & \multicolumn{2}{c}{ Perda dentária } & $\boldsymbol{p}=\mathbf{0 , 1 7 6}$ \\
\hline & Sim $(\%)$ & Não $(\%)$ & Total \\
\hline Sexo & & & \\
Masculino & $6(10,2)$ & $23(39,0)$ & $29(49,2)$ \\
Feminino & $11(18,6)$ & $19(32,2)$ & $30(50,8)$ \\
\hline Total & $17(28,8)$ & $42(71,2)$ & $59(100)$ \\
\hline
\end{tabular}

Com relação à existência de correlação entre perda dentária e a frequência de escovação, diferenças estatisticamente significantes não foram evidenciadas (Tabela 3 ).

Tabela 3 - Associação entre perda dentária e frequência de escovação entre os estudantes da Escola Monsenhor Linhares. Fortaleza (CE), maio de 2019.

\begin{tabular}{|c|c|c|c|}
\hline \multirow[t]{2}{*}{ Variáveis } & \multicolumn{2}{|c|}{ Perda dentária } & \multirow{2}{*}{$\begin{array}{c}p=0,245 \\
\text { Total }(\%)\end{array}$} \\
\hline & $\operatorname{Sim}(\%)$ & Não (\%) & \\
\hline Frequência de escovação & & & \\
\hline Duas vezes por dia & $3(5,1)$ & $14(23,7)$ & $17(28,8)$ \\
\hline Três vezes por dia & $13(22)$ & $21(35,6)$ & $34(57,6)$ \\
\hline Acima de três vezes por dia & 0 & $4(6,8)$ & $4(6,8)$ \\
\hline Não tem regularidade & $1(1,7)$ & $3(5,1)$ & $4(6,8)$ \\
\hline Total & $17(28,8)$ & $42(71,2)$ & $59(100)$ \\
\hline
\end{tabular}

Tabela 4 - Associação entre perda dentária e visita ao dentista entre os estudantes da Escola Monsenhor Linhares. Fortaleza (CE), maio de 2019.

\begin{tabular}{|c|c|c|c|}
\hline \multirow[t]{2}{*}{ Variáveis } & \multicolumn{2}{|c|}{ Perda dentária } & \multirow{2}{*}{$\frac{\mathrm{p}=\mathbf{0 , 0 2 7}}{\text { Total }(\%)}$} \\
\hline & $\operatorname{Sim}(\%)$ & Não (\%) & \\
\hline Visita ao dentista & & & \\
\hline Sim & $17(28,8)$ & $32(54,2)$ & $49(83)$ \\
\hline Não & 0 & $10(17)$ & $10(17)$ \\
\hline Total & $17(28,8)$ & $42(71,2)$ & $59(100)$ \\
\hline
\end{tabular}

Verificou-se, entretanto, associação estatisticamente significante $(p=0,027)$ entre perda dentária e visita ao cirurgião-dentista, como demonstrado na Tabela 4. 
Ao se verificar associações entre visita ao dentista e variáveis como sexo $(p=0,184)$ e etnia $(p=0,463)$, não foram observadas diferenças estatisticamente significantes. Ademais, não houve correlação entre perda dentária e as variáveis uso do fio dental $(p=0,258)$, e "frequência do uso do fio dental $(p=0,512)$.

\section{DISCUSSÃO}

No Brasil, a perda dentária entre adolescentes distribui-se de forma desigual, de acordo com as regiões e os grupos sociais. Em todos os levantamentos epidemiológicos realizados, em âmbito nacional, houve desvantagem para as Regiões Norte, Nordeste e Centro-Oeste ${ }^{10}$. Barbato e Peres ${ }^{11}$, ressaltam a associação entre a inexistência de água fluorada na região nordeste e as perdas dentárias. No tocante ao estado do Ceará, Xavier e colaboradores $^{12}$ chamam atenção para o fato de que $82,43 \%$ dos municípios analisados em seu estudo receberem água fluoretada em níveis classificados como satisfatórios, atendendo aos padrões estabelecidos na legislação vigente.

Não obstante se tratar de um problema de saúde pública, a perda dentária tem sido pouco investigada entre adolescentes brasileiros ${ }^{9}$. Os resultados do presente estudo demonstraram uma prevalência de perdas dentárias de $28,8 \%$, percentual superior àquele apontado no levantamento epidemiológico nacional de 2010 (17,4\%). Por questões metodológicas (estudo transversal), não se pôde testar hipóteses, entretanto, este elevado percentual, entre os estudantes da escola municipal, pode ser fruto da falta de acesso aos serviços de saúde bucal. Contrapondo-se a estes resultados, Melo e colaboradores ${ }^{13}$ e Silveira e colaboradores ${ }^{9}$ apontaram, em seus estudos, índices próximos aos do levantamento nacional: 17,2 e $16,3 \%$, respectivamente.

Na série histórica brasileira, pode se observar uma tendência de decréscimo da prevalência de perdas dentárias por meio dos levantamentos epidemiológicos nacionais. Em 1986 e 2003, as médias de dentes perdidos por adolescente foram 1,2 e 0,9 , respectivamente ${ }^{14,15}$. Na presente pesquisa,
0,51 dentes perdidos foi a média obtida entre os adolescentes, tendo sido inferior à do último levantamento epidemiológico nacional. Enfatiza-se, pois, o fato de, apesar de os participantes deste estudo terem apresentado maior prevalência de perda dentária, comparada ao levantamento nacional, a média de dentes perdidos mostrou-se inferior e variando de uma a sete unidades dentárias, o que sugere uma heterogeneidade entre os alunos no tocante aos aspectos relacionados à saúde bucal ou mesmo ao acesso aos serviços de odontologia.

De acordo com Peres e colaboradores ${ }^{16}$, as perdas dentárias entre adolescentes e adultos constatadas no último levantamento se devem, possivelmente, ao somatório de alguns fatores, como a melhoria das condições socioeconômicas (em especial da educação) e do sistema de saúde, a exemplo da exposição à fluoretação de águas e da expansão do uso de dentifrícios fluoretados. Essas duas medidas resultaram em grande cobertura populacional nas décadas de 1980 e 1990 e explicam, em grande parte, a redução da prevalência e extensão da cárie dentária no Brasil, principal causa de perda dentária.

Não se observou no presente estudo associação estatisticamente significante entre a perda dentária e o sexo dos adolescentes $(p=0,176)$, ao contrário de outras pesquisas, em que se constatouse maior prevalência de perdas no sexo feminino ${ }^{11,15,17}$. Embora pareça um contrassenso, uma hipótese para tal diferença, citada nessas pesquisas, foi a maior utilização de serviços odontológicos por mulheres, induzida por motivos estéticos ou por cuidados com a saúde. Acredita-se que o excesso de procedimentos invasivos, dentro de um modelo curativo-restaurador, pode se constituir em um fator importante para a perda dentária, com a tendência a uma superexposição de tratamento invasivo, ou à exodontia, caso não exista condição para um procedimento especializado de maior complexidade $^{13,18}$.

Neste estudo, o segundo molar permanente superior do lado direito (dente 17) foi o dente mais prevalente no tocante à perda dentária, resultado 
este diferente de outros estudos, que apontam o primeiro molar inferior permanente como o dente mais afetado por perdas dentárias em adolescentes ${ }^{11,13,19}$. Dentre as hipóteses citadas por esses autores para explicar a maior prevalência de perdas de primeiros molares estão: a dificuldade de higienização, devido à localização e as condições favoráveis ao acúmulo de placa. $\mathrm{O}$ fato de o segundo molar ter sido mais afetado por perda dentária que o primeiro molar, no presente estudo, pode ter ocorrido devido ao seu período de erupção ser um dos últimos da fase de transição da pré-adolescência para adolescência, ou seja, a transição dos cuidados maternos para o autocuidado.

Corroborando outros estudos ${ }^{9,13,20}$ na presente pesquisa não houve associação entre a perda dentária e a frequência de escovação $(p=0,245)$. Observa-se que, apesar de 57,6\% dos estudantes escovarem os dentes três vezes ao dia, $22 \%$ sofreram perda dentária. Esse alto percentual pode estar associado aos maus hábitos alimentares, bem como à má qualidade da escovação. Ressalta-se, aqui, a importância da educação em saúde no processo de formação de hábitos alimentares adequados a uma boa saúde bucal e geral ${ }^{21}$. Nesse sentido, programas de prevenção em saúde bucal voltados para adolescentes e suas famílias deveriam ser implementados de forma contínua.

Constatou-se que mais de $80 \%$ dos estudantes já tinham ido ao consultório de dentista, entretanto, dada a elevada prevalência de perdas dentárias, pode não ter havido regularidade nessas visitas. Por outro lado, Ely e colaboradores ${ }^{22}$ consideram que a expansão do acesso ao serviço odontológico pode, em contrapartida, resultar no aumento de procedimentos mutiladores.

Em virtude das restrições no acesso aos serviços odontológicos especializados no âmbito do Sistema Único de Saúde (SUS), em especial para a realização de tratamentos endodônticos, a extração pode se configurar como um desfecho inevitável, sobretudo quando a cárie se encontra em estágio avançado de destruição tecidual, especialmente para os grupos de menor renda ${ }^{5}$. Costa e colaboradores ${ }^{10}$ ressaltam que, aparentemente, não faltam recursos para o acesso a serviços odontológicos quando se trata de extração dentária, comparando-se a outros procedimentos. Bulgareli e colaboradores ${ }^{23}$ destacam a dificuldade de preservação dos dentes no âmbito dos serviços odontológicos, mesmo entre os mais jovens.

A prevalência da perda dentária também foi objeto de investigação de Tôrres e colaboradores ${ }^{24}$, que analisaram a influência de fatores socioeconômicos e demográficos, o uso de serviço odontológico e a dor, nos últimos seis meses, a partir dos dados do Inquérito de Saúde Oral de São Paulo de 2008, em adolescentes de 15 a 19 anos de idade. As variáveis independentes que mostraram associação com a perda dentária foram: cárie, dor de dente, baixa escolaridade do pai e renda familiar inferior a meio salário-mínimo per capita. Os resultados sugerem que os fatores socioeconômicos podem contribuir para o aumento da perda dentária precoce entre adolescentes.

A política nacional de saúde preconiza a universalidade do acesso como um princípio do Sistema Único de Saúde (SUS), porém, a universalidade do acesso aos serviços de saúde bucal ainda parece ser uma realidade difícil de se alcançar. Ainda que a saúde bucal tenha sua importância reconhecida, uma parcela importante da população brasileira não tem acesso a serviços odontológicos. Apesar de os escolares terem sido alvo das políticas de saúde bucal durante décadas, dados do levantamento epidemiológico do Ministério da Saúde - SB Brasil 2003, revelaram que $13 \%$ dos jovens brasileiros entre 15 e 19 anos de idade nunca haviam realizado uma visita ao cirurgião-dentista ${ }^{25,26}$.

Visando à prevenção de perdas dentárias na adolescência, Spezia ${ }^{27}$ recomenda a implementação de políticas públicas efetivas, haja vista essas perdas ocasionarem por si só gastos elevados com reabilitação oral para a saúde pública. $\mathrm{O}$ autor ressalta a importância do tratamento preventivo, para a melhoria da qualidade de vida desses indivíduos, em detrimento da valorização do tratamento curativo ou reabilitador, por meio de próteses. 
Devido ao reduzido tamanho da amostra e ao seu desenho, os resultados deste estudo não podem ser generalizados, o que se constitui em uma limitação, entretanto, estudos de prevalência de perda dentária devem ser estimulados, por contribuírem para fomentar o debate sobre o tema e fornecer subsídios para a implementação de políticas públicas direcionadas a diferentes públicos-alvo.

\section{CONSIDERAÇÕES FINAIS}

A prevalência de perda dentária entre os adolescentes participantes deste estudo mostrou-se elevada, comparada aos resultados do último levantamento epidemiológico nacional, o que remete à necessidade de se implementarem políticas públicas de saúde que contribuam para modificar esta realidade.

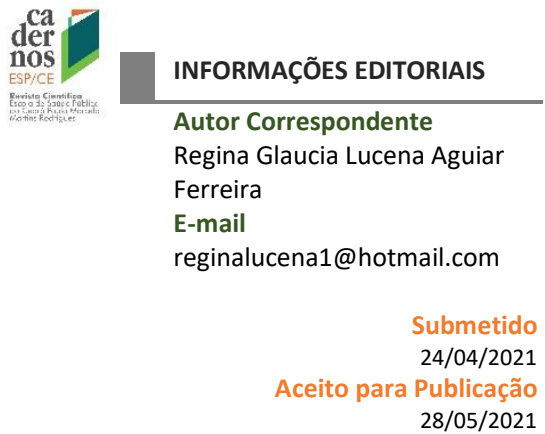

\section{REFERÊNCIAS}

1. Oliveira DC, Pereira PN, Ferreira FM, Paiva SM, Fraiz FC. Impacto relatado das alterações bucais na qualidade de vida de adolescentes: revisão sistemática. Pesqui Bras Odontopediatria Clin Integr. 2013;13(1):123-9. https://doi.org/ 10.4034/PBOCI.2013.131.18.

2. Peres, KG, Cascaes AM, Leão, ATT, Côrtes MIS, Vettore MV. Aspectos sociodemográficos e clínicos da qualidade de vida relacionada à saúde bucal em adolescentes. Rev saúde pública. 2013; 47(Supl 3):19-28. https://10.1590/S00348910.2013047004361.

3. Vingilis ER, Wade TJ, Seeley JS. Predictors of Adolescent Self-rated Health Analysis of the National Population Health Survey. Can J Public Health. 2002; 93(2):193-7. https://doi:10.1007/BF03404999.

4. Garcia AFG, Sobrinho, JEL, Araujo JC, Menezes VA, Costa EMMB. Influência do fator socioeconômico no comportamento dos adolescentes em relação à saúde bucal. Revista Odonto. 2008; 31:53-61. http://dx.doi.org/10.15603/2176-1000.

5. Ministério da Saúde (BR), Secretaria de Atenção à Saúde, Secretaria de Vigilância em Saúde. SB Brasil 2010: Pesquisa Nacional de Saúde Bucal: resultados principais. Brasília: Ministério da Saúde, 2012.

6. Frias AC, Antunes JLF, Junqueira SR, Narvai PC. Determinantes individuais e contextuais da prevalência de cárie dentária não tratada no Brasil. Rev Panam Salud Publica. 2007; 22(4): 279-285.

7. Narvai PC, Frazão P, Roncalli AG, Antunes JLF. Cárie dentária no Brasil: declínio, polarização, iniqüidade e exclusão social. Rev Panam Salud Publica. 2006; 19(6): 385-393.

8. World Health Organization. The World Oral Health Report 2003. Continuous Improvement of Oral Health in the 21st Century

- the Approach of the WHO Global Oral Health Programme. Genebra: Organização Mundial de Saúde; 2003.

9. Silveira MF, Freire RS, Nepomuceno MO, Martins MEBL, Marcopito LF. Cárie dentária e fatores associados entre adolescentes no norte do estado de Minas Gerais, Brasil: uma análise hierarquizada. Ciênc Saúde Coletiva. 2015; 20(11):335164. https://doi.org/10.1590/1413-812320152011.12262014.

10. Costa, SM, Abreu MHNG, Vasconcelos M, Lima RCGS, Verdi M, Ferreira EF. Inequalities in the distribution of dental caries in Brazil: a bioethical approach. Cienc Saude Colet. 2013; 18: 461-70.

11. Barbato PR, Peres MA. Tooth loss and associated factors in adolescents: a Brazilian population based oral health survey. Rev Saúde Pública. 2009; 43(1):13-25. https://doi.org/10.1590/S0034-89102009000100003.

12. Xavier CO, Costa MTP, Fraga ACA, Burgoa MIR, Almeida LC, Vasconcelos MP, Costa, TES. Análise do flúor no sistema de abastecimento do Ceará. Cadernos ESP. 2019;13(2):12-23.

13. Melo FGC, Cavalcanti AL, Fontes LBC, Granville-Garcia AF, Cavalcanti SDALB. Perda precoce de molares permanentes e fatores associados em escolares de 9, 12 e 15 anos da rede pública municipal de Campina Grande, Estado da Paraíba, Brasil. Acta Scientiarum Health Sciences. 2011;33(1):99-105. https://doi.org/ 10.4025. 
14. Ministério da Saúde, Centro de Documentação. Levantamento epidemiológico em saúde bucal: Brasil, zona urbana, 1986. Brasília: Ministério da Saúde, 1988.

15. Barbato PR, Nagano HCM, Zanchet FN, Boing AF, Peres MA. Perdas dentárias e fatores sociais, demográficos e de serviços associados em adultos brasileiros: uma análise dos dados do Estudo Epidemiológico Nacional (Projeto SB Brasil 2002-2003). Cad Saude Publica. 2007; 23(8):1803-14. https://doi.org/10.1590/S0102-311X2007000800007.

16. Peres MA, Barbato PR, Reis, SMGB, Freitas CHSM, Antunes, JLF. Perdas dentárias no Brasil: análise da Pesquisa Nacional de Saúde Bucal 2010. Rev. Saúde Pública. 2013; 47(3):78-89. https://doi.org/10.1590/S0034-8910.2013047004226.

17. Pattussi MP, Peres KG, Boing AF, Peres MA, Da Costa JSD. Self-rated oral health and associated factors in Brazilian elders. Community Dent Oral Epidemiol. 2010; 38: 348-59. https:doi.org/10.1111/j.1600-0528.2010.00542

18. Lopez R, Baelum V. Gender. differences in tooth loss among Chilean adolescents: Socio-economic and behavioral correlates. Acta Odontol Scand. 2006; 64(3):169-76.

19. Normando D, Cavacami C. The influence of bilateral lower first permanent molar loss on dentofacial morphology-a cephalometric study. Dent Press J Orthod. 2010; 15(6):100-6. https://doi.org/10.1590/S2176-94512010000600013.

20. Colussi PRG. Perdas dentárias, fatores associados e seu impacto sobre a qualidade de vida em adolescentes de Passo Fundo/RS [Tese] Passo Fundo: Universidade Federal do Rio Grande do Sul, 2013.

21. Freire MCM. Dieta, Saúde Bucal e Saúde Geral. In: Buischi YP. Promoção de Saúde Bucal na Clínica Odontológica. São Paulo: Artes Médicas/EAP-APCD; 2000. p. 275.

22. Ely HC, Abegg C, Celeste RK, Pattussi MP. Impacto das equipes de saúde bucal da Estratégia da Saúde da Família na saúde bucal de adolescentes do sul do Brasil. Ciênc Saúde Coletiva. 2016; 21(5):1607-16. https://doi.org/10.1590/141381232015215.07822015.

23. Bulgareli JV, Faria ET, Cortellazzi KL, Guerra LM, Meneghim MC, Ambrosano GMB, Frias AC, Pereira AC. Fatores que influenciam o impacto da saúde bucal nas atividades diárias de adolescentes, adultos e idosos. Rev. Saúde Pública. 2018; 52:44:1-9. https://doi.org/10.11606/s1518-8787.2018052000042.

24. Tôrres LHN, Zanatta aj, apizolato RA, Grillo CM, Frias AC, Sousa MLR. Reasons related to tooth loss among adolescents in São Paulo, Brazil. Braz J Oral Sci. 2014; 13(1): 37-42. https://doi.org/10.1590/1677-3225

25. Barros AJD, Bertoldi AD. Desigualdades na utilização e no acesso a serviços odontológicos: uma avaliação em nível nacional. Ciênc Saúde Coletiva. 2002; 7(4): 709-17. https://doi.org/10.1590/S1413-81232002000400008.

26. Baldani MH, AC, Brito WH, Lawder, Mendes YBESilva FFM, Antunes JLF. Determinantes individuais da utilização recente de serviços odontológicos por adolescentes e adultos jovens de baixa renda. Rev bras epidemiol. 2010. 13(1): 160-62. https://doi.org/10.1590/S1415-790X2010000100014

27. Spezzia S. Perdas dentárias na adolescência. Revista Odontológica de Araçatuba. 2016; 37(3):9-1 